\title{
Norois
}

Environnement, aménagement, société

La Loire. Sociétés, risques, paysages, environnement

\section{La vulnérabilité des hommes et des habitations face au risque d'inondation dans le Val nantais (1841-2003)}

Entre législation nationale et pratiques locales

Vulnerability of populations and buildings during floods in the Val nantais

(Loire River valley): a gap between local politics and national laws (1841-2003)

Valérie Jousseaume, Julie Landrein et Denis Mercier

\section{(2) OpenEdition}

Journals

\section{Édition électronique}

URL : http://journals.openedition.org/norois/866

DOI : $10.4000 /$ norois. 866

ISBN : 978-2-7535-1540-6

ISSN : $1760-8546$

\section{Éditeur}

Presses universitaires de Rennes

Édition imprimée

Date de publication : 1 septembre 2004

Pagination : 29-45

ISBN : 978-2-7535-0054-9

ISSN : 0029-182X

\section{Référence électronique}

Valérie Jousseaume, Julie Landrein et Denis Mercier, «La vulnérabilité des hommes et des habitations face au risque d'inondation dans le Val nantais (1841-2003) », Norois [En ligne], 192 | 2004/3, mis en ligne le 20 août 2008, consulté le 01 mai 2019. URL : http://journals.openedition.org/norois/866 ; DOI $10.4000 /$ norois.866

Ce document a été généré automatiquement le 1 mai 2019.

(c) Tous droits réservés 


\section{La vulnérabilité des hommes et des habitations face au risque d'inondation dans le Val nantais (1841-2003)}

Entre législation nationale et pratiques locales

Vulnerability of populations and buildings during floods in the Val nantais

(Loire River valley): a gap between local politics and national laws (1841-2003)

Valérie Jousseaume, Julie Landrein et Denis Mercier

\section{NOTE DE L'ÉDITEUR}

Cet article a été reçu le 7 avril 2004 et définitivement accepté le 25 juin 2004.

1 Le risque résulte de la rencontre, sur un même espace, d'un aléa et d'une vulnérabilité. Pour le risque d'inondation de plaine, il s'agit d'un aléa hydro-météorologique (des pluies cumulatives le plus souvent) à l'origine de crues importantes entraînant un débordement hors du lit mineur ou une remontée de nappe, et une inondation. Ce phénomène aléatoire est récurrent et, chaque année, la France connaît des crues et des inondations. Il est difficile de montrer des tendances à l'aggravation de l'aléa hydro-météorologique et de possibles relations avec les changements climatiques contemporains. En revanche, il est plus aisé de mesurer l'accroissement de la vulnérabilité qui est le fruit de modifications de l'occupation humaine du territoire. Selon la loi $n^{\circ}$ 95-101 du 2 février 1995, la vulnérabilité peut se définir comme « l'existence de biens et d'activités pouvant subir des dommages et de personnes pouvant subir des préjudices ».

Des études pléthoriques ont été menées en France sur la cindynique en général et sur les inondations en particulier. Les espaces méditerranéens, gravement touchés ces dernières années ont fait l'objet d'études minutieuses (Lemartinel, 2001 ; Vinet, 2003). Les crues et inondations de plaine ont également été étudiées, le long de la Saône (Astrade, 1996, 
2002 ; Pottier, 2002), de la Marne (Pottier, 1998), de la Seine (Bravard, 2000 ; Guillon, 2003). Depuis longtemps, la Loire et ses crues ont également intéressé les géographes (Dion, 1934, 1961 ; Fénelon, 1971 ; Jeanneau, 1984 ; Oudot, 1989 ; Archambault, 1995 ; Dacharry, 1996 ; Schulé, 2000 ; Giraudet et Ménanteau, 2001 ; Rode, 2001). Des études techniques ont évalué la vulnérabilité en Loire moyenne entre Nevers et Angers, où l'on ne compte pas moins de 240 communes, 115000 logements, 290000 personnes, 13600 entreprises, 1600 équipements collectifs en zone inondable. Cependant, jusqu'ici rien n'a été publié sur l'accroissement de la vulnérabilité en aval d'Angers.

Le Val nantais, célèbre pour le dynamisme de son bassin maraîcher, se situe aux portes de l'agglomération nantaise en pleine croissance démographique, et est en proie à l'étalement urbain (fig. 1). L'étude minutieuse à l'échelle des deux communes principales $\mathrm{du}$ Val nantais, La Chapelle-Basse-Mer et Saint-Julien-de-Concelles, permet de quantifier l'évolution de la vulnérabilité dans cette zone inondable depuis la construction, au milieu $\mathrm{du} \mathrm{XIX}^{\mathrm{e}}$ siècle, de la levée qui le protège. Quelles sont les méthodes pour évaluer ces métamorphoses? Quand et comment s'est opéré l'accroissement de la vulnérabilité dans le Val nantais ? Y a-t-il cohérence entre politiques municipales et politiques nationales? Telles sont les questions qui structurent cet article.

Figure 1 : Localisation du Val nantais Study area

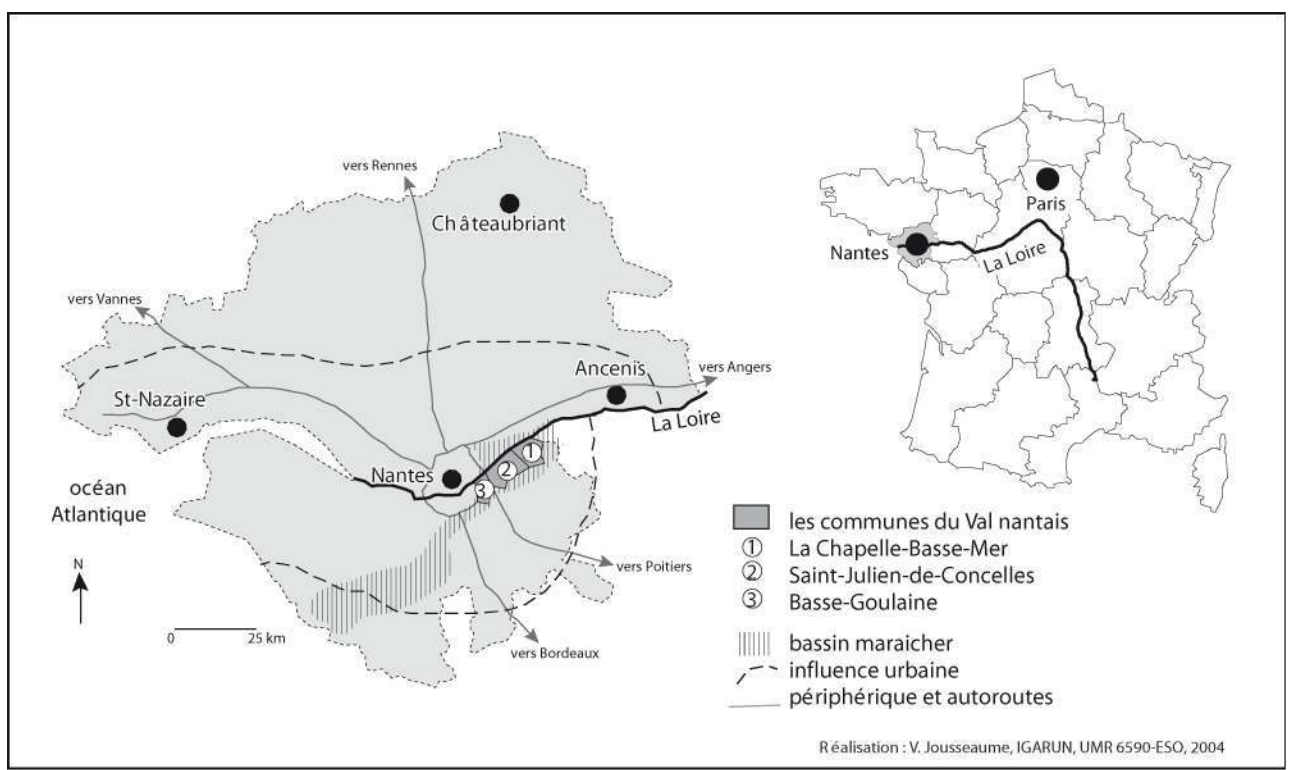

\section{La zone d'étude}

Le Val nantais correspond à une dépression topographique délimitée par des coteaux escarpés. Suite à l'effondrement tectonique plio-quaternaire de ce morceau de socle, la Loire a occupé l'ensemble de la dépression, la comblant de ses alluvions après des millénaires d'une dynamique de tressage (Burnouf et al., 2003). La microtopographie du Val nantais résulte de cet héritage hydrosédimentaire. Les hauteurs décroissent logiquement de l'amont du val (alt. sup. à 7,5 m NGF au lieu-dit la Haute-Vallée) vers l'aval (alt. inf. à $5 \mathrm{~m}$ à Saint-Julien-de-Concelles). Les altitudes décroissent également depuis les points hauts du bourrelet de berge le long de la rive gauche du bras principal 
(alt. sup. à 7,5 m entre le Moulin-de-l'̂̂le et la Chebuette), vers les points les plus bas qui se concentrent au pied du coteau méridional, jadis parcouru par un bras secondaire de la Loire (alt. de $6 \mathrm{~m}$ à La Chapelle-Basse-Mer, entre 5 et $4 \mathrm{~m}$ à Saint-Julien-de-Concelles). La méthode hydrogéomorphologique (Masson et al., 1996), associant cote des plus hautes eaux connues, analyse de terrain, photo-interprétation, mémoire des crues (Mercier, 2004), permet une délimitation aisée de la zone inondable du Val nantais (fig. 2).

Figure 2 : La topographie du val nantais Topography of Val nantais

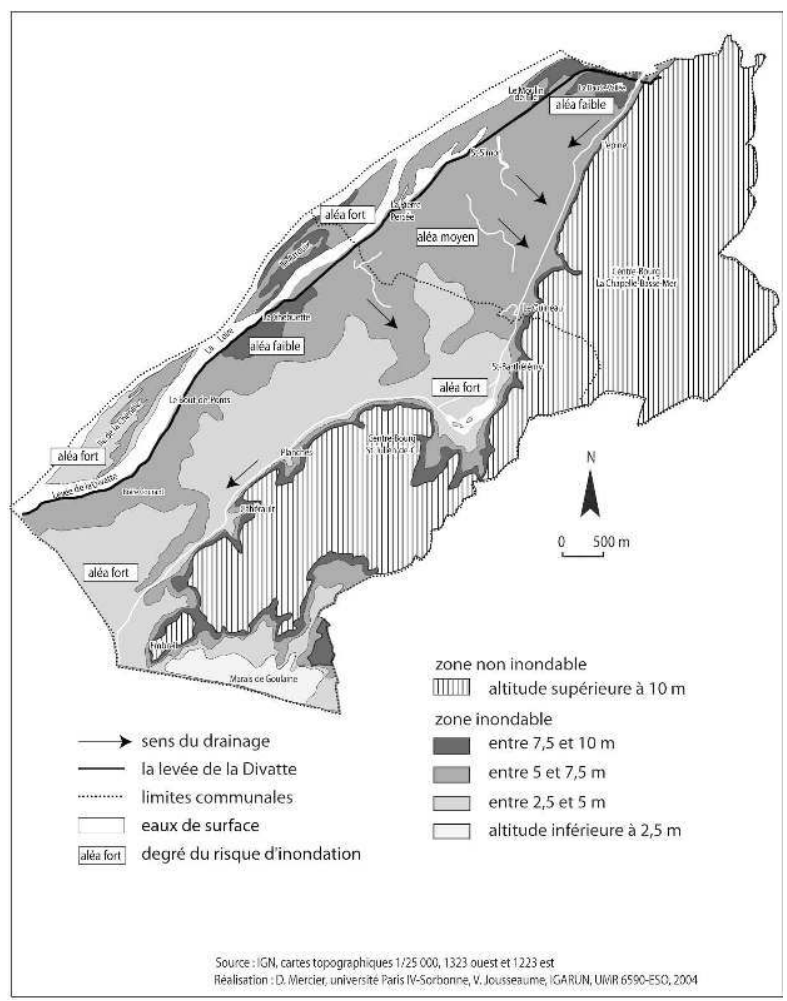

5 La levée de la Divatte, érigée sur le bourrelet de berge entre 1847 et 1856, est la dernière des digues construites le long de la Loire (Marion, 1981). Cette dernière atteint la cote de $10 \mathrm{~m}$ NGF à La Chapelle-Basse-Mer et $9 \mathrm{~m}$ à Saint-Julien-de-Concelles, plus un muret d'une cinquantaine de centimètres. Avant cet aménagement tardif, le fleuve débordait régulièrement, inondant son lit majeur (fig.3). La Loire aval connaît ses crues généralement en hiver (décembre à mars) associées à des précipitations océaniques. Cependant, les crues de printemps (juin 1856) ne sont pas inconnues et correspondent à des crues mixtes associant pluies océaniques abondantes et précipitations méditerranéennes de forte intensité sur la partie amont du bassin versant. Beaucoup plus exceptionnelles sont les crues d'été comme celle de juillet 1783. Depuis la construction de la levée, deux alternatives s'offrent en cas de crue, soit les eaux sont contenues par la digue et les inondations affectent seulement les points bas de la vallée par résurgence de la nappe $(1995,1982,1936 \ldots)$, soit la digue cède ou est submergée et l'inondation ennoie tout le val. La levée de la Divatte a déjà cédé deux fois, lors des crues de juin 1856 et de décembre 1910, date référence des plus hautes eaux connues avec une cote de crue à 9,40 $m$ NGF. Le val fut alors immergé sous un à quatre mètres d'eau durant de nombreux jours.

Comment mesurer l'évolution de la vulnérabilité de cette zone inondable? 
Figure 3 : Les principales crues de la Loire dans le Val nantais depuis le XVIII e siècle Main floods of Loire River since 18th century in the Val nantais

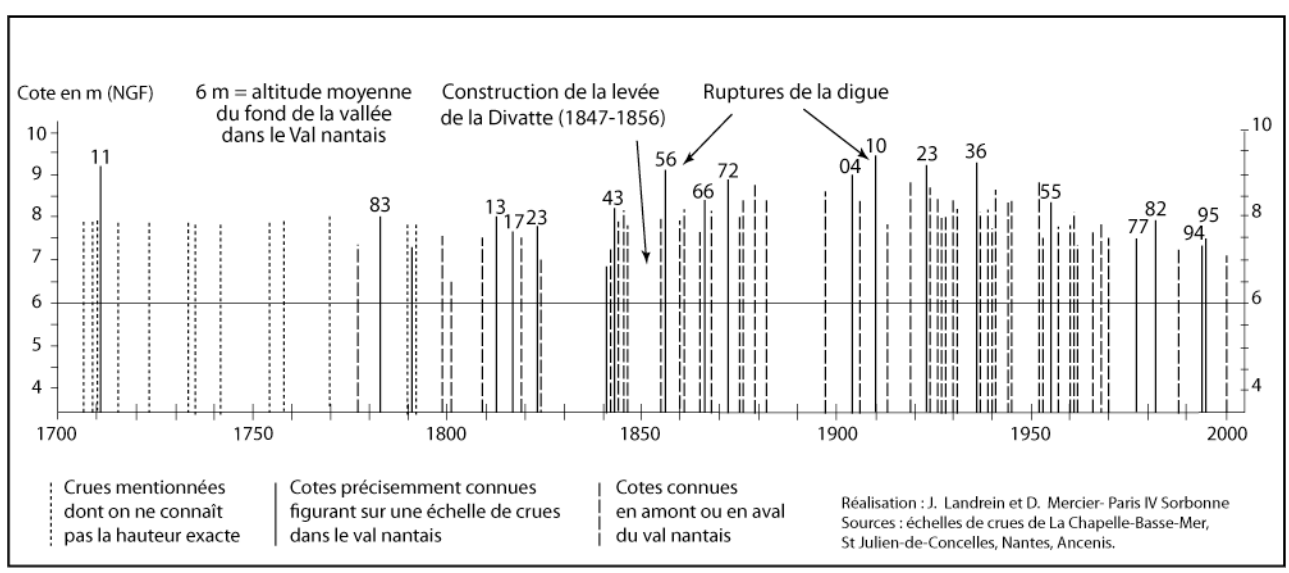

\section{Les méthodes}

7 Les échelles de publication de l'INSEE, à la commune ou à l'IRIS ${ }^{1}$, ne permettent pas d'appréhender la question de la vulnérabilité des personnes et des habitations dans le Val nantais. La première raison est que le risque d'inondation ne concerne pas l'ensemble des surfaces communales. La Chapelle-Basse-Mer et Saint-Julien-de-Concelles sont de vastes communes, respectivement 2214 et 3174 hectares. Les surfaces communales se répartissent comme suit : à La Chapelle-Basse-Mer le lit mineur occupe environ 300 ha, la vallée 750 ha et le plateau 1150 ha, à Saint-Julien-de-Concelles le lit mineur concerne 330 ha, la vallée 1640 ha et le marais de Goulaine 270 ha, le plateau 950 ha. La seconde raison est que le peuplement demeure extrêmement diffus. En effet, l'habitat y est dispersé en plus de 160 lieux-dits habités par commune, comptant de 2 à 230 habitants en dehors des bourg$^{2}$. En 1999, le bourg de La Chapelle-Basse-Mer ne groupe que 1450 des 4272 Chapelains et celui de Saint-Julien-de-Concelles seulement 2087 des 6203 Concellois, soit le tiers des populations communales. Pour répondre à la problématique de la vulnérabilité face au risque d'inondation dans le Val nantais, une observation infracommunale fine est donc nécessaire.

8 Pour ce faire, les listes nominatives des recensements généraux de la population (RGP) ont été dépouillées, afin de répartir les populations par lieux-dits. Les RGP antérieurs à la Seconde Guerre mondiale sont aux archives départementales. Au-delà de 30 ans, l'INSEE verse ses RGP aux archives départementales, cela concerne à ce jour ceux de 1946, 1954, 1962 et 1968. Néanmoins, pour des raisons matérielles, les archives de Loire-Atlantique ne conservent que quelques communes, comme cela est le cas pour La Chapelle-Basse-Mer. Les RGP non conservés par les archives, sont normalement confiés aux mairies, ce qui n'était pas le cas pour le RGP 1968 de Saint-Julien-de-Concelles. Pour la période récente, l'analyse n'aurait pu être menée sans les documents déposés par les agents recenseurs communaux en 1968 et en 1999. En effet, par chance, des récapitulatifs laissaient apparaître des bilans de personnes et de résidences, avec les lieux-dits écrits en marge.

9 Six dates ont été retenues: 1999, 1968, 1936, 1906, 1876 et 1841. Ces dates, au rythme trentenaire, bornent les différentes phases démographiques des deux communes étudiées. En effet, le RGP de 1841 pose la situation de l'occupation du Val nantais juste 
avant la construction de la digue protectrice. La population croît par un bilan naturel excédentaire jusqu'au RGP de 1876 qui marque le maximum démographique rural du canton. À partir de cette date, la population décline fortement par exode rural jusqu'en 1906, puis stagne jusqu'en 1936. De 1841 à 1936, la croissance puis le déclin s'opèrent sans apport migratoire extérieur. De la Seconde Guerre mondiale jusqu'au RGP de 1968, les deux communes connaissent un fort développement de leur activité légumière. Le moteur de la croissance démographique demeure essentiellement endogène. Enfin, depuis la construction du pont de Bellevue sur la Loire, en 1974, les communes de Saint-Julien-deConcelles et La Chapelle-Basse-Mer sont marquées par le mouvement de périurbanisation nantais. Des populations nouvelles arrivent, le plus souvent sans attache, ni communale, ni agricole.

La Chapelle-Basse-Mer et Saint-Julien-de-Concelles permettent donc une observation de l'évolution de la vulnérabilité face au risque d'inondation, dans des contextes démographiques fort contrastés dans le temps.

\section{Les résultats : un accroissement important et récent de la vulnérabilité}

\section{Un doublement de la population exposée depuis 1936, essentiellement à Saint-Julien-de-Concelles}

Au RGP de 1999, la population habitant la zone inondable du Val nantais s'élève à 2715 personnes à Saint-Julien-de-Concelles et 1225 personnes à La Chapelle-Basse-Mer, soit 3940 personnes au total. L'occupation de la vallée et des pourtours du marais de Goulaine est ancienne puisqu'en 1841 déjà, la zone inondable comptait 2996 habitants (fig. 4).

Figure 4 : Évolution de la population dans le Val nantais Evolution of the population in the Val nantais

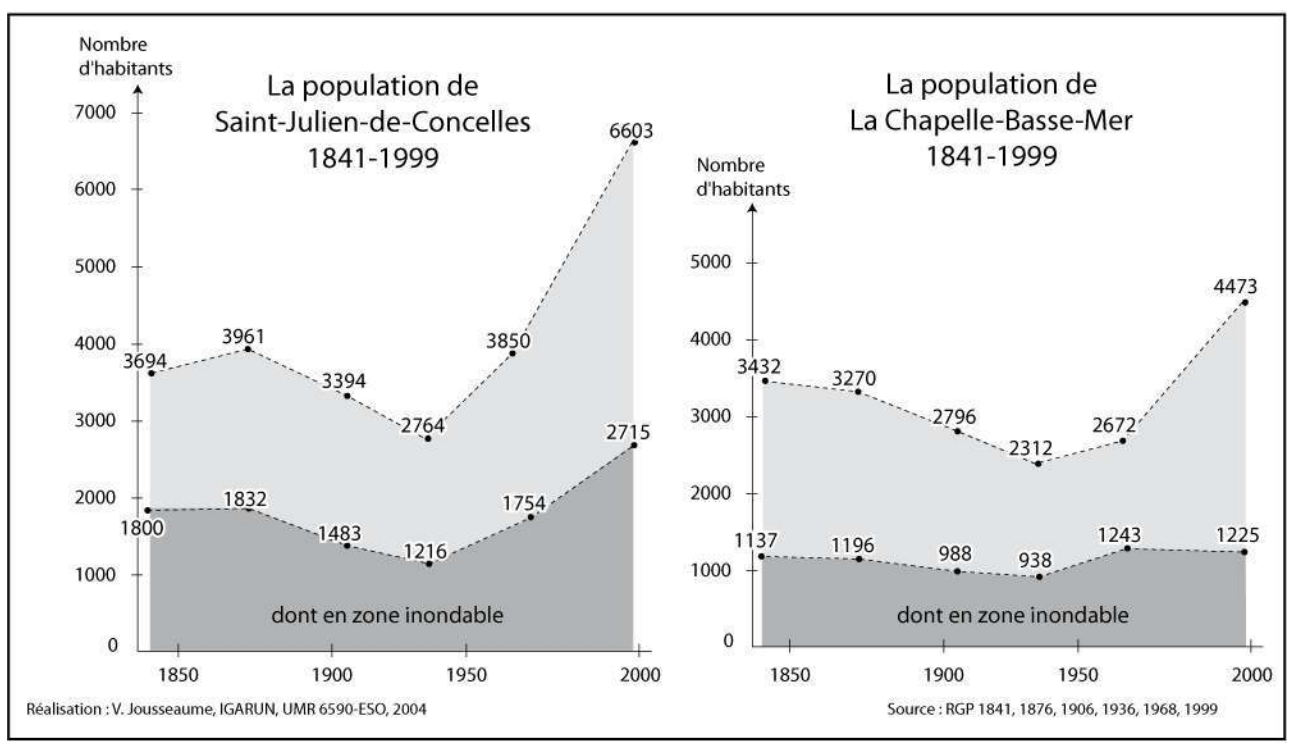

Entre 1841 et 1876, dans un contexte de croissance démographique, la construction de la Levée de la Divatte n'a pas entraîné une progression de la population dans le val. Le paradoxe n'est qu'apparent puisque la motivation de la construction de la digue n'a 
jamais été la protection des personnes et des habitations. Son objectif était la protection des terres agricoles. Au début du XIx ${ }^{e}$ siècle, l'essentiel de la vallée était composé de "communs", propriétés collectives de l'ensemble des habitants de la commune. Les cartes anciennes dessinent une prairie humide, voire marécageuse, sans haie. Au milieu $\mathrm{du} \mathrm{XIX}^{\mathrm{e}}$ siècle en Loire-Atlantique, les progrès techniques et surtout les forts revenus de la rente foncière ont poussé la bourgeoisie à demander le partage et l'appropriation privée des « communs » (Bourrigaud, 1994). Dans le Val nantais comme ailleurs, le partage eut lieu après de difficiles procès pour déterminer les ayants droit. Le syndicat des propriétaires du marais de Goulaine fut créé en 1830 et celui du marais du Chêne en 1846, dans le but d'assécher les marais pour les cultiver et les premiers travaux de drainage furent entrepris. La même année fut créée l'association syndicale de la Divatte regroupant les propriétaires du Val nantais, afin d'ériger une levée, financée pour partie seulement par l'État. Sur le bourrelet de berge et à l'amont de la vallée, les terres mieux drainées (alt. sup. à $7 \mathrm{~m}$ NGF) autour des villages furent mises en culture. Les villages agricoles se sont quelque peu développés, mais cette croissance démographique fut annihilée par le déclin des villages de mariniers, dont l'activité était malmenée par l'essor du transport ferroviaire. La production commerciale de légumes frais pour le marché urbain (asperges, petits pois, haricots verts), de matière première pour l'industrie nantaise (osier, chanvre pour la corderie et la voilerie, légumes à conserve, fruits pour les biscuiteries) s'est développée. Les points bas, plus humides, furent transformés en prés pour les animaux, enclos dans un bocage à maille fine. Ce paysage perdurera jusqu'au remembrement en 1971 (Jousseaume et al., 2003).

13 De 1876 à 1936, l'exode rural a entraîné la perte de 30 \% de la population dans chacune des deux communes de l'étude. La zone inondable a connu un déclin de même ordre. En 1936, lors du minimum démographique des deux communes, la population du val était alors de 2154 personnes, c'est-à-dire 938 individus à La Chapelle-Basse-Mer et 1216 à Saint-Julien-de-Concelles. La vulnérabilité des personnes connaît là son minimum.

$\mathrm{Au}$ milieu du $\mathrm{xx}^{\mathrm{e}}$ siècle eut lieu une translation vers l'est de la ceinture maraîchère nantaise, sous l'effet de l'urbanisation progressive des terres maraîchères de la commune de Nantes. Les « cultivateurs » du Val nantais, comme ils se nommaient eux-mêmes, sont devenus alors les nouveaux " maraîchers nantais ». Progressivement, les agriculteurs de la vallée se sont organisés pour dépasser les obstacles à leur spécialisation: l'eau, la commercialisation et le parcellaire, ce faisant ils poursuivirent la transformation du milieu. Ainsi, en 1955, l'Association syndicale des maraîchers de la Divatte fut créée, afin d'améliorer le drainage des eaux par le creusement de profonds fossés vers l'ancien bras de pied de coteau, devenu le canal du Champ Blond, et de construire une prise d'eau en Loire afin d'assurer une irrigation collective à faible coût. En 1961, la coopérative du Val nantais fut fondée afin d'assurer une commercialisation commune des légumes. En 1971, le remembrement débuta. Les prairies humides et le bocage disparurent, laissant place à un paysage maraîcher de champs ouverts et géométriques. Le marais du Chêne, devenu propriété de la commune de Saint-Julien-de-Concelles, fut drainé et transformé en un plan d'eau associé à un espace de loisirs. Cet assèchement fut parachevé en 1990, quand, suite à l'inondation par remontée de la nappe de 1982, le SIVOM Loire-et-Goulaine mit en place une station de huit pompes à Basse-Goulaine pour accélérer le drainage et limiter les effets de résurgence des hautes eaux en vallée. Alors que, par le passé, les fossés se remplissaient d'eau en hiver, les variations du niveau de la Loire ne sont plus perceptibles par les habitants de la vallée en 2004. Le maraîchage occupe aujourd'hui tous les terrains 
dont l'altitude est supérieure à $5 \mathrm{~m}$ NGF, soit un gain de $2 \mathrm{~m}$ d'altitude en un siècle. L'activité maraîchère a transformé une prairie humide en champs de légumes, en évacuant toute eau de surface.

Figure 5 : Évolution du nombre des habitations dans le Val nantais Evolution of the number of inhabitations of the Val nantais

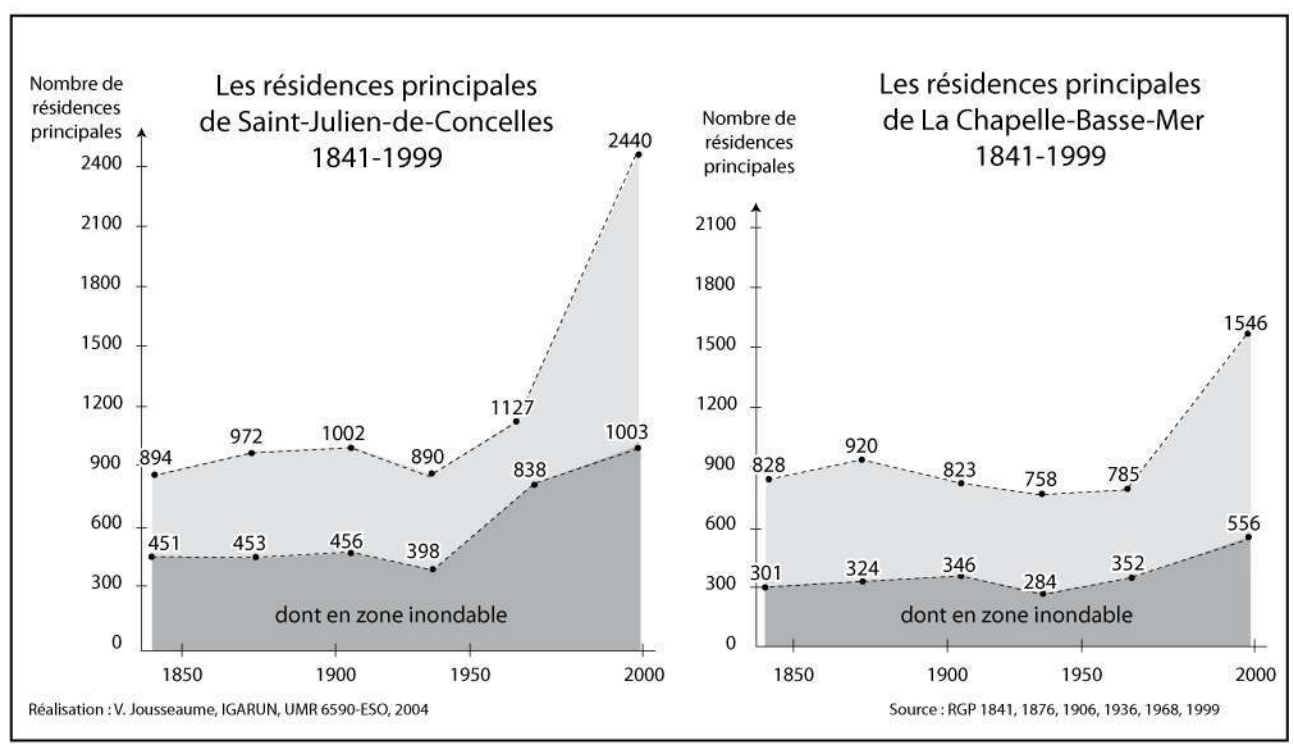

De 1936 à 1968, dans le contexte de cette mutation agricole, la vallée de La ChapelleBasse-Mer a connu une croissance de 287 personnes pendant que le reste de la commune stagnait. À Saint-Julien-de-Concelles, la population de la vallée s'est accrue de 538 personnes. En 1968, la zone inondable retrouve exactement le niveau de population de 1841.

À partir de la décennie 1980, la mécanisation du maraîchage entraîne une diminution des besoins de main-d'œuvre. L'agriculture n'est donc plus le moteur de l'accroissement de la population dans la vallée, le relais est pris par la périurbanisation. Le mouvement périurbain nantais atteint les communes étudiées à partir du milieu des années 1970 . Ainsi, entre les RGP de 1968 et 1999, la population chapelaine totale s'est accrue de 1600 habitants, soit une croissance annuelle moyenne de $1,52 \%$ et la population concelloise, avec 2400 habitants en plus, a enregistré une évolution annuelle de 1,58\% essentiellement liée au bilan migratoire. Le fait le plus remarquable, au cours de cette période de forte croissance exogène, est la dissociation des évolutions démographiques de la vallée, entre les deux communes (fig. 4). Ainsi, le val de Saint-Julien-de-Concelles a accueilli 960 habitants supplémentaires entre 1968 et 1999, absorbant $40 \%$ de la croissance communale. Entre le minimum démographique de 1936 et 1999, la population concelloise en zone inondable a plus que doublé passant de 1216 à 2715 habitants, soit une augmentation de 1500 personnes. Entre 1968 et 1999, la population de la vallée a légèrement diminué sur la commune de La Chapelle-Basse-Mer. La vallée qui groupait $46,5 \%$ de la population chapelaine en 1968 , ne représente plus que $27 \%$ de la population communale en 1999. Depuis 1968, la commune de Saint-Julien-de-Concelles participe donc seule à l'accroissement de la vulnérabilité de la population face au risque d'inondation dans le Val nantais (fig. 6). 
L'accroissement de la vulnérabilité des personnes n'est que modeste au vu de l'accroissement de la vulnérabilité des biens, en particulier des habitations (fig. 6).

Figure 6 : Évolution de la vulnérabilité de la zone inondable du Val nantais entre 1841 et 1999 Evolution of the vulnerability of the Val nantais flood area (1841-1999)

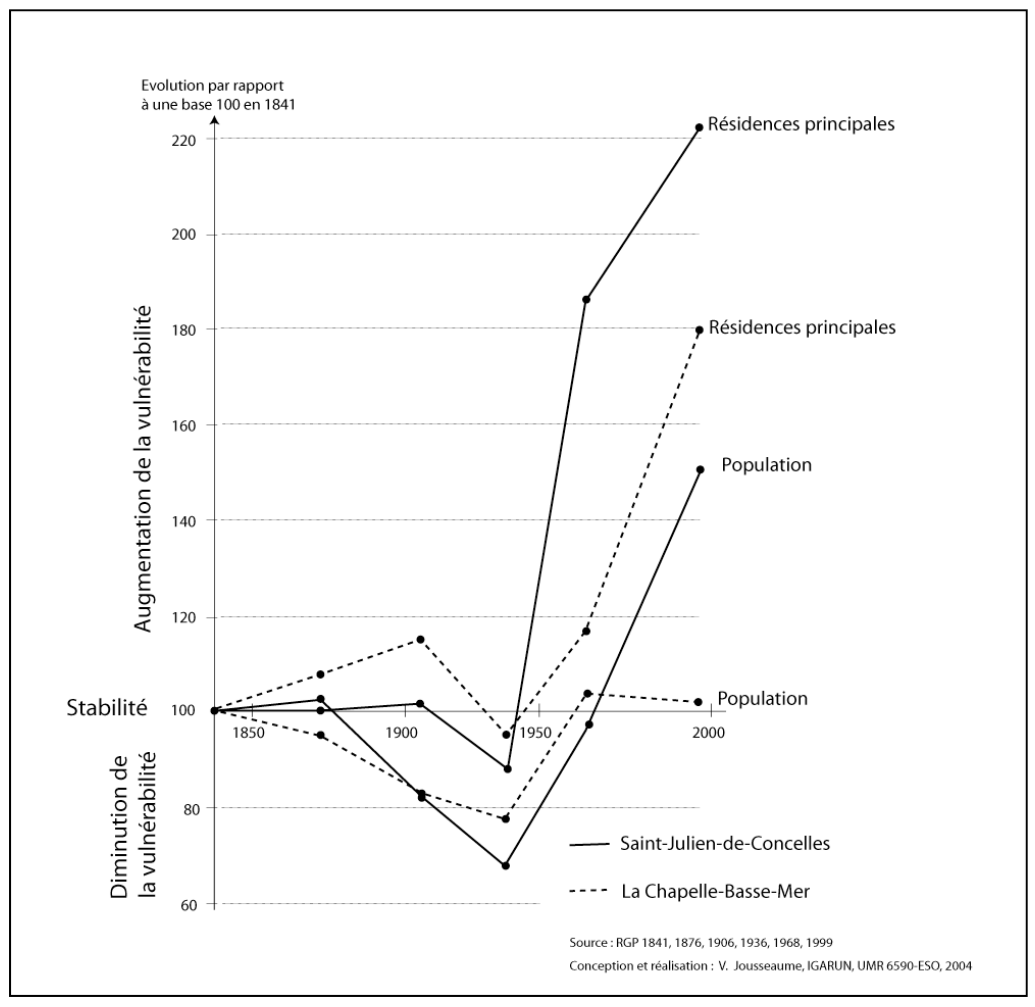

\section{Des habitations de plus en plus nombreuses et de plus en plus vulnérables}

En 1841, le peuplement du Val nantais s'organisait en deux lignes parallèles, parfaitement identiques. Sur le bas-coteau, au pied duquel coulait un bras secondaire de la Loire (souligné par des lieux-dits aux noms évocateurs : Port-Moron, Port-Biry, Port-Égaud, Basse-Rivière, le Gué-au-Voyer), l'alignement continu des villages agricoles de quelques habitations était rythmé par de gros villages diversifiés tels que l'Épine, le Guineau, SaintBarthélémy, le bourg de Saint-Julien (100 maisons et 367 habitants en 1841), Cahérault jusqu'au village d'Embreil qui contrôlait un gué entre le marais de Goulaine et la vallée de la Loire. Ces lieux-dits étaient tous implantés à une altitude de 9,5 m NGF, qui correspond bien à la limite légale des plus hautes eaux connues $(9,40 \mathrm{~m})$. Au bord du fleuve, la même organisation se répétait. Sur le bourrelet de berge s'alignaient tous les deux kilomètres, d'importants villages de pêcheurs, de mariniers, d'artisans et de commerçants, tels que Saint-Simon, la Pierre-Percée, la Chebuette, le Haut-Village (actuel Bout-des-Ponts) et Boire-Courant, qui comptaient chacun entre 30 et 80 maisons pour 100 et 250 habitants au $\mathrm{XIX}^{\mathrm{e}}$ siècle. Entre ceux-ci, de petits villages de 2 à 10 maisons pour 10 à 40 personnes, groupaient de façon très imbriquée plusieurs borderies, petites exploitations agricoles en faire-valoir direct. Le centre de la vallée, dont l'altitude est inférieure à 6 m NGF, n'était pas habité en 1841. 

phases, qui correspondent à des travaux de drainage importants. Vers les années 1860, après la construction de la digue, quelques villages furent créés dans les points bas: Sébastopol, Malakoff, le Claray, les Divettes, le Petit-Pont, le Mortier. Au tournant de la décennie 1970, au moment du remembrement, quelques lieux-dits sont nés encore, de la construction isolée de maisons neuves, souvent liées à l'activité maraîchère: la Grenouillère, le Mortier des Places, le Quai-Bondu, l'Ouche-Brégeon, l'Ouche-Blé, Le PâtisDoré.

Pour l'essentiel, on peut dire que la structure de peuplement du Val nantais décrite en 1841, liée à un système d'exploitation et à un système de propriété aujourd'hui révolus, perdure jusqu'en 2004 (fig. 7).

Figure 7 : La population du val nantais par lieu-dit (1968-1999) Val nantais population by locality (1968-1999)

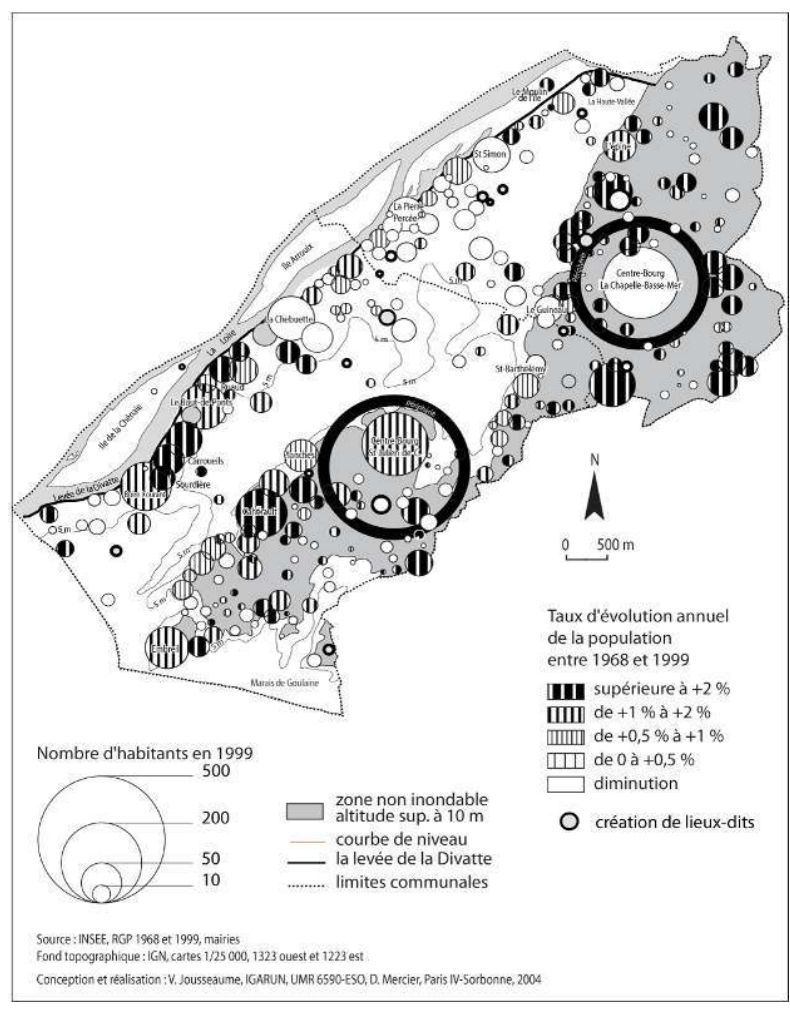

21 Cette permanence de la forme générale du peuplement est liée à la persistance du bâti, même dans un contexte de déclin démographique. Ainsi, la perte d'un tiers de la population entre 1876 et 1936 n'a donné lieu qu'à une réduction de $12 \%$ du nombre de résidences principales (fig. 5), car les ménages, composés de 4 personnes en moyenne en 1841, ne comptaient plus que 3 individus en 1936. Les résidences principales abandonnées sont devenues des logements vacants ou des résidences secondaires. Nous ne disposons pas de données avant la Seconde Guerre mondiale, mais au RGP de 1968 on dénombre encore à Saint-Julien-de-Concelles 80 logements vacants et 195 résidences secondaires dans la vallée, soit un quart du parc immobilier! Les résidences secondaires se localisaient en bordure de Loire. De la fin du XIX ${ }^{e}$ siècle à la décennie 1960, sur les plages de sable, la baignade et la pêche étaient des loisirs appréciés de la population nantaise. L'accès était facile, puisque la levée de la Divatte fut ouverte à la circulation des voitures. 
À partir de 1971 avec le remembrement et l'intensification maraîchère d'une part, la destruction de seuils rocheux et l'accélération du courant du fleuve d'autre part, le développement de la circulation automobile et l'intégration progressive dans l'aire nantaise enfin, ont ôté tout caractère touristique au Val nantais. Ces modestes résidences secondaires ont pu retourner partiellement dans le logement principal. Mais, les structures d'exploitation et de propriété passées expliquent une vacance forte. Les " valliats ", comme ils se nommaient eux-mêmes, étaient des bordiers, c'est-à-dire des paysans micro-propriétaires qui vivaient groupés en village. En dehors de quelques belles maisons ligériennes de négociants situées dans les villages ayant une activité fluviale, le patrimoine bâti ancien de la vallée était extrêmement modeste, imbriqué dans le bâti voisin, sans jardin, inadapté aux normes du confort de la seconde moitié du $\mathrm{xx}^{\mathrm{e}}$ siècle. C'est pourquoi, ces anciennes borderies sont très souvent devenues des garages ou des dépendances.

Dans le contexte de croissance démographique, la permanence de la forme générale du peuplement masque un fort développement de constructions nouvelles et un étalement des villages qui entraîne aujourd'hui leur coalescence.

Le renouveau de la construction dans la vallée a débuté plus précocement à Saint-Juliende-Concelles, où entre 1936 et 1968, le nombre de résidences principales a doublé en vallée et la population moyenne des ménages s'est abaissée à deux personnes (fig. 5). Dans le même temps, à La Chapelle-Basse-Mer, la progression de la population a partiellement été absorbée par une croissance du nombre moyen d'individus par ménage, qui est remonté à 3,5 personnes en 1968. À cette époque, en effet, les ouvriers agricoles étaient des adolescents, venus des marches de Bretagne, pour trouver un emploi avant leur service militaire. Ces jeunes hommes étaient la plupart du temps logés par leur employeur. Cette différence de structure des ménages entre les deux communes laisse à penser que, dès cette époque, la croissance de l'habitat dans le val à Saint-Julien-deConcelles n'est pas exclusivement liée à l'essor de l'agriculture.

Entre 1968 et 1990, les deux communes participent à part égale, à l'accroissement du nombre des habitations exposées au risque d'inondation. Le boom de la construction nouvelle dans la vallée de La Chapelle-Basse-Mer eut lieu entre 1965 et 1978 (Landrein, 2003). Il correspond à l'arrivée à l'âge adulte d'une génération nombreuse et une période faste du maraîchage. Aussi, beaucoup parmi les enfants de maraîchers ont assuré la succession de leur père. Avec la démocratisation de l'automobile, d'autres, travaillant hors de la commune, ont choisi de faire construire sur un terrain familial. C'est à la fin des années 1970 qu'un ralentissement de la construction neuve en vallée apparaît à La Chapelle-Basse-Mer, celle-ci devient presque nulle à partir de la fin des années 1980.

Depuis cette période, une importante divergence de pratiques urbanistiques peut être observée entre les deux communes (Landrein, 2003 ; Meunier, 2003). Les travaux en cours de F.-A. Le Guillou (2004) confirment l'importance de la construction neuve en vallée à Saint-Julien-de-Concelles. Ici, la proximité du bourg avec la zone inondable a également favorisé le développement des infrastructures municipales (salles de sport, salle polyvalente, centre socioculturel) dans le val, sur les terres voisines du marais du Chêne, devenues propriété communale lors du remembrement.

26 Au-delà du simple nombre, le type des maisons est lui aussi de plus en plus vulnérable. En effet, avant la Seconde Guerre mondiale, les maisons étaient adaptées au risque d'inondation, puisque l'habitat était soit sur cave en terre battue, soit sur tertre naturel 
ou artificiel. Au cours des décennies 1960 et 1970, conformément aux exigences légales de l'époque, les habitations neuves étaient construites sur garage, l'habitat étant réservé à l'étage. Curieusement, depuis vingt ans, les habitations neuves sont de plain-pied, l'étage lorsqu'il existe ne compte que les chambres. En cas d'inondation, c'est donc la quasi totalité du bien immobilier qui serait sinistrée. Une analyse détaillée de l'accroissement de la vulnérabilité architecturale fera l'objet d'un article ultérieur, actuellement en préparation.

La figure 6 fait la synthèse de l'évolution de la vulnérabilité des personnes et des habitations dans le Val nantais. Après un siècle de stagnation, le dernier demi-siècle a connu une croissance des habitations de $80 \%$ à La Chapelle-Basse-Mer et $120 \%$ à SaintJulien-de-Concelles.

\section{Discussion : les différentes échelles de l'intérêt général}

La législation nationale sur les risques naturels s'est considérablement renforcée ces deux dernières décennies. Ayant pris conscience de la faible efficacité des mesures structurelles pour lutter contre les aléas des cours d'eau (les barrages et les digues ne suppriment pas définitivement les risques d'inondation, sur la Loire comme ailleurs), l'action de l'État privilégie désormais la limitation de la vulnérabilité des zones inondables. La première action en ce sens fut la création par la loi du 13 juillet 1982, des Plans d'Exposition aux Risques Naturels Prévisibles. Ils prévoyaient une réglementation de l'occupation du sol et des constructions dans les zones à risque (Plan des Surfaces Submersibles), mais restèrent sans grand succès. Il faut attendre la loi du 2 février 1995 et l'instauration des Plans de Prévention des Risques (PPR) pour entrevoir un changement notoire au niveau national pour la limitation de la vulnérabilité. Le PPR du Val nantais fut approuvé le 12 mars 2001, soit six années après la promulgation de la loi, durée utilisée par les communes concernées (La Chapelle-Basse-Mer, Saint-Julien-de-Concelles et BasseGoulaine) et les services de l'État pour négocier les modalités d'applications des contraintes d'urbanisme. Tout récemment, la loi du 30 juillet 2003 relative à la prévention des risques, vise à développer une culture du risque. Les communes sont désormais tenues d'organiser une réunion publique tous les deux ans (article 40), elles doivent inventorier, entretenir, et protéger les marques des crues historiques (article 42). Enfin, toute personne doit être informée du caractère inondable du bien, au moment des transactions immobilières (article 77).

Dans ce cadre législatif, comment expliquer les différences communales de l'accroissement de la vulnérabilité du Val nantais?

\section{Convergences et divergences d'intérêts entre particuliers et collectivités}

Dans le contexte de développement agricole, les agriculteurs, qui formaient également l'essentiel des propriétaires, ont pris en charge les transformations du milieu, par la constitution d'associations syndicales pour la construction de la digue, l'assèchement, l'irrigation. La convergence d'intérêts avec les communes paraissait néanmoins totale et un transfert partiel de la prise en charge des aménagements de la sphère privée agricole 
vers les collectivités locales s'est opéré dans le dernier tiers $\mathrm{du} \mathrm{xx}^{\mathrm{e}}$ siècle. Les maires des deux communes et le conseiller général du canton étaient des maraîchers ou avaient des liens professionnels directs avec le maraîchage jusqu'en 1983. L'agriculture concernait directement plus de la moitié de la population résidente en 1954. En 1990, l'emploi agricole représentait encore $40 \%$ de l'emploi communal et à peine moins d'un quart en 1999, sans compter le reste de la sphère maraîchère. C'est pourquoi, les municipalités chapelaine et concelloise ont soutenu le remembrement en 1971. Dès ce moment, le conseil général de Loire-Atlantique a contribué aux travaux d'entretien de la digue. Et, en 1987, celui-ci reprend totalement à sa charge la levée de la Divatte, dont le syndicat propriétaire et gestionnaire était financièrement incapable d'assurer l'entretien. Depuis, le Département et la Région ont investi 15 millions d'euros dans la réfection de la digue. Enfin, les syndicats de propriétaires se sont dissous dans un vaste SIVOM Loire-etGoulaine en 1989. Ainsi, le drainage des eaux de la vallée, le fonctionnement des pompes de Basse-Goulaine, ne sont plus aujourd'hui que partiellement financées par les propriétaires fonciers et immobiliers de la vallée, l'essentiel est pris en charge par les collectivités locales adhérentes du SIVOM.

Depuis la fin de la décennie 1970 et le développement du mouvement de périurbanisation, les intérêts sont devenus contradictoires dans la vallée. On peut distinguer trois tendances, en se gardant de tout manichéisme puisque ces positions sont souvent entremêlées au sein des familles ou des conseils municipaux. D'un côté, le morcellement du parcellaire et le morcellement de la propriété en vallée, hérités du système agraire passé, sont favorables aux spéculations individuelles en faveur de l'urbanisation. En effet, le prix du terrain constructible, de l'ordre de 75 euros le mètre carré, est sans commune mesure avec le prix du terrain agricole, pour lequel d'ailleurs il n'existe pas de marché (aujourd'hui les maraîchers louent les terres, mais ne souhaitent que rarement les acheter). De l'autre côté, les agriculteurs en activité redoutent un mitage de leur espace de production. Certains maraîchers en appellent à la délimitation d'une zone agricole exclusive, au même titre qu'existent des zones industrielles, afin de coordonner l'actuel développement de serres sous forme de grands tunnels en plastique, et ainsi éviter tous les conflits paysagers que le voisinage de telles structures pourraient engendrer. Ce mitage est d'autant plus craint, que la mécanisation pousse à l'agrandissement. Cette faim de terre provoque dans la partie amont de la vallée qui est étroite, la progression du maraîchage sur le coteau. Enfin, et plus récemment, les partisans de la valorisation paysagère et récréative de la Loire et de la levée de la Divatte, renouant avec une fonction ancienne disparue, organisent des randonnées, des courses, le dimanche annuel sans voiture sur la levée, accueillent l'escale d'animations ligériennes telle que «la remontée du sel de Guérande à Saumur »...

Dans ce contexte, les deux municipalités, de même tendance politique, développent des stratégies communales totalement différentes; le point commun reste que les idées de valorisation écologique, paysagère, récréative ou touristique des bords de Loire restent peu entendues.

La municipalité de La Chapelle-Basse-Mer considère que le maintien de l'activité agricole est l'intérêt communal. Celle-ci entretient en effet 350 emplois directs en 1999 et une centaine d'emplois indirects. Les maires de la commune ont presque toujours eu un lien direct avec le maraîchage ; l'actuel maire, élu depuis 1989, était le chef d'une entreprise d'extraction de sable de Loire pour les maraîchers. Le POS, dès sa création en 1978, a limité le mitage résidentiel de l'espace maraîcher et a réduit la construction dans la 
vallée. D'ailleurs, du vote de la loi instaurant les PPR en 1995 jusqu'à la validation du PPR du Val nantais en 2001, la négociation de la municipalité de La Chapelle-Basse-Mer avec les services de l'État chargés de le mettre en œuvre, a porté sur un assouplissement des restrictions en faveur du développement de l'activité maraîchère en vallée. Ainsi, des constructions neuves peuvent être autorisées si elles concernent des bâtiments agricoles (serres, hangars) et des maisons d'agriculteurs à proximité immédiate du siège d'exploitation.

La municipalité de Saint-Julien-de-Concelles, dont les maires qui changent à chaque mandat depuis 20 ans, sont employés de banque ou commerçants, a progressivement restreint sa défense des intérêts agricoles pour développer une politique explicitement tournée vers l'accueil résidentiel, y compris en vallée ${ }^{3}$ (fig. 7). La municipalité de SaintJulien-de-Concelles a négocié âprement, avec la préfecture de Loire-Atlantique, un assouplissement du PPR entre 1995 et 2001, et s'enorgueillit d'avoir «sauvé » (sic) (de l'interdiction de bâtir) une trentaine de terrains en fond de vallée et d'avoir ôté de la définition de la zone inondable des secteurs de pied de coteau, par la transformation de la norme légale des plus hautes eaux connues (PHEC) soit 9,4 m (crue de 1910), en plus hautes eaux prévisibles (PHEP) soit 8,5 m pour définir le zonage du PPR. C'est dans l'un de ces secteurs inondables, que la municipalité concelloise projette la construction, très contestée, d'un supermarché de $2500 \mathrm{~m}^{2}$. De même, l'ensemble sportif s'étend encore en zone inondable, par la construction d'une salle de tennis de table d'un coût de 870000 euros. Cette salle devra accueillir des pongistes concellois mais également ceux d'une commune voisine du plateau. À travers ces deux projets, on peut se poser la question du rôle de l'intercommunalité. De plus, face à l'accroissement du flux des migrations pendulaires et des camions sur la digue, une route fut construite dans le val en 2003, joignant le bourg de Saint-Julien-de-Concelles au périphérique nantais, pour une somme de plus de 16 millions d'euros. La création de cette route départementale conséquence de l'urbanisation, rétroagit pour en devenir une cause. La construction neuve s'est maintenue dans le val, particulièrement le long du bourrelet de berge au Ruaud, Les Carroueils, La Sourdière notamment (fig. 7). L'agriculture est repoussée vers les terres les plus basses, les plus humides, les moins favorables à la production légumière de plein champ. Le maraîchage concellois évolue donc vers de la production hors sol sur laine de roche, souvent sous serres de verre chauffées. La vulnérabilité économique de la filière maraîchère s'accroît donc également beaucoup plus à Saint-Julien-de-Concelles qu'à La Chapelle-Basse-Mer; alors même que dans le cas de la production hors sol, la qualité agronomique des sols ne justifie plus une localisation dans le lit majeur du fleuve (Joyeux, 2004).

Cette divergence récente de choix politiques des deux conseils municipaux, protection du maraîchage à La Chapelle-Basse-Mer et accueil résidentiel à Saint-Julien-de-Concelles, explique la dissociation des évolutions de la vulnérabilité des personnes et des biens face au risque d'inondation dans le Val nantais. Mais la question de la limitation de la vulnérabilité face au risque d'inondation, considérée comme l'intérêt général à l'échelle nationale, n'est nullement perçue à l'échelle de ces deux communes. 


\section{Convergences et divergences d'intérêts entre l'échelon local et l'échelon national} val, n'est pas exposée à un risque mortel. En effet, la Loire aval, contrairement au sud de la France, ne connait pas d'inondation brutale. Les ondes de crue sont connues et annoncées plusieurs jours à l'avance (réseau CRISTAL de Météo-France, services hydrométriques des DIREN). Les populations ont le temps d'être évacuées. Même en cas de rupture de la digue, comme en 1910, la montée des eaux dans le val s'étire sur plus de deux jours. Les municipalités auraient cependant à faire face à une épineuse question : où loger les 3940 personnes de la zone inondable? Les infrastructures publiques de SaintJulien-de-Concelles, susceptibles d'héberger les sinistrés, se situent presque toutes en zone inondable, à $5 \mathrm{~m}$ d'altitude. Or dans le cadre de la gestion des territoires inondables, l'anticipation demeure souvent un atout précieux pour la bonne efficacité des secours (Varagne, 2003).

39 Ces deux arguments n'ont aucune validité si on change d'échelles tant spatiales, que temporelles.

Premièrement, l'abaissement de la ligne d'eau du fleuve, si elle demeure sans effet majeur à l'échelon local, est d'un coût énorme aux échelles régionale et nationale. Outre les modifications écologiques de l'estuaire, la remontée de la salinité a nécessité un déplacement vers l'amont de la prise d'eau potable de la ville de Nantes. Plus largement, l'incision du lit a nécessité l'enrochement de kilomètres de berges, fort coûteux pour la collectivité tenue de protéger la propriété privée, peu esthétique et totalement inefficace sur le moyen terme. Surtout, cela a entraîné une fragilisation de la base des ouvrages d'art de l'ensemble de la Loire: ponts, quais, digues (y compris la levée de la Divatte). L'élément révélateur fut l'effondrement du pont de Tours en 1978, date marquant le tournant de la politique nationale (Bravard, 2000). Depuis, les extractions de sable de Loire ont été limitées puis interdites totalement en 1995. Dans le cadre du plan national Loire Grandeur Nature en œuvre depuis 1994, la reconstruction des seuils rocheux, détruits il y a 30 ans, à commencé entre Nantes et Angers : un épi à radier a été érigé au Fresne-sur-Loire en 2003, et celle du seuil rocheux de Bellevue à proximité immédiate du Val nantais, est en projet ${ }^{5}$ (Ginsburger-Vogel, 2004). De même, face aux coûts du dragage 
du chenal et de l'adaptation de la hauteur des ponts, l'interruption de la navigation marchande de gros tonnage entre Saint-Nazaire et Nantes est envisagée. La conjugaison de ces actions est favorable à une ré-élévation de la ligne d'eau de la Loire à moyen ou long terme, comme nous l'enseigne l'étude de la résilience de tout hydrosystème. Ces faits ne peuvent être ignorés dans la perspective d'un aménagement durable du territoire du Val nantais. Mais là encore, tout manichéisme entre commune et État est à éviter, puisque la transformation de la cote des plus hautes eaux connues en plus hautes eaux prévisibles dans le PPR du Val nantais a été validée par la préfecture de la Loire-Atlantique.

Secondement, le danger de mort n'est nullement le motif de l'action de l'État contre la progression de la vulnérabilité des zones inondables. À l'échelle nationale, c'est la vulnérabilité des biens (habitations, entreprises agricoles, industrielles ou commerciales, infrastructures publiques) qui coûte cher à la collectivité. Les inondations représentent 80 \% du coût total des catastrophes naturelles en France. Et la facture ne cesse de croître, selon la caisse centrale de réassurance, elle représente un coût total pour la France de 4,8 milliards d'euros entre 1982 et 2002, chiffre qu'il faudrait doubler selon F. Vinet (2003) en comptant les dommages aux biens publics non assurés (routes, infrastructures...), les dommages agricoles et les pertes restées à la charge des particuliers.

42 À l'échelle locale, seuls sont perçus et mesurés les bénéfices individuels et collectifs de l'occupation de la vallée à court terme : le risque d'une inondation est totalement renié et les arguments utilisés valorisent exclusivement la création de richesses issues de l'occupation du val ${ }^{6}$. À l'échelle nationale, se pose la question de la collectivisation des coûts de cette prise de risque.

\section{Conclusion}

43 Le Val nantais appartient au lit majeur de la Loire. Cette zone inondable a été protégée par une levée pour devenir une prairie bocagère au xix siècle. Tout au long du xxe siècle, il fut draîné, asséché, remembré, jusqu'à former, à partir de la décennie 1960, le cœur d'un important bassin de production maraîcher. Enfin, sous les effets de la périurbanisation nantaise, la vallée connaît une pression récente à l'urbanisation. Dans ce contexte, si la vulnérabilité des personnes a stagné à La Chapelle-Basse-Mer, elle a doublé à Saint-Julien-de-Concelles depuis 1936. En quelques décennies, le nombre des habitations en zone inondable a progressé de 80 \% à La Chapelle-Basse-Mer et de $120 \%$ à Saint-Juliende-Concelles. De plus, l'habitat, dont l'essentiel du logement familial est au rez-dechaussée, est de moins en moins adapté au risque d'inondation. La question n'est pas de faire table rase du processus historique d'aménagement du Val nantais. En revanche, dans un contexte de périurbanisation, on peut se demander si cette zone inondable peut devenir un espace d'urbanisation comme les autres? De l'agriculture aux loisirs, de nombreuses alternatives de développement existent, favorables aux intérêts locaux aussi bien que nationaux, favorables à une relation durable entre la Loire et la société qui la borde.

Cet exemple illustre les distorsions, entre d'une part un intérêt général national qui est double, diminuer le coût de la vulnérabilité des biens en zone inondable et préserver le patrimoine naturel, et un intérêt général communal qui est, pour partie, la mutualisation d'intérêts particuliers convergents, en faveur de la protection du bassin maraîcher à La 
Chapelle-Basse-Mer, en faveur de l'urbanisation du val à Saint-Julien-de-Concelles. Ce cas illustre également l'abîme entre le temps long de la nature et le temps court du politique.

\section{BIBLIOGRAPHIE}

ARchambault (M.), 1995. - « Propositions pour la protection des vals de Loire contre les crues dévastatrices du fleuve », Norois, vol. 42, n 166, p. 305-318.

ASTRADE (L.), 1996. - La Saône en crue : dynamique d'un hydrosystème anthropisé, thèse de Doctorat, université Paris 4 Sorbonne, 358 p.

-, 2002. - « Les crues et les inondations de la Saône », dans J.-P. Bravard, J. Combier, N. Commerçon (dir.), La Saône, axe de civilisation, Lyon, Presses universitaires de Lyon, p. 157-171. BOURRIGAUD (R.), 1994. - Le développement agricole au XIXe siècle en Loire-Atlantique, Nantes, CDMOT, $496 \mathrm{p}$.

BRAVARD (J.-P.) (dir.), 2000. - Les régions françaises face aux extrêmes hydrologiques. Gestion des excès et de la pénurie, Paris, Sedes, $287 \mathrm{p}$.

BuRnouf (J.), CARCAUd (N.), GARCIN (M.), 2003. - « La Loire entre mythes et réalités », Nantes, 303 Arts, Recherches et Créations, $\mathrm{n}^{\circ} 75$ spécial La Loire, p. 13-17.

CHARRIER (P.), 1997. - La vallée de la Loire des Ponts-de-Cé à Bellevue (entre Anjou et Pays nantais): évolutions et sensibilités, thèse de Doctorat, Université de Nantes, $411 \mathrm{p}$.

DACHARRY (M.), 1996. - « Les grandes crues historiques de la Loire », La Houille Blanche, nº 6-7, p. 47-53.

Dion (R.), 1934. - Le Val de Loire, étude de géographie régionale, Tours, Arrault et $\mathrm{C}^{\mathrm{ie}}, 752 \mathrm{p}$.

-, 1961. - Histoire des levées de la Loire, Paris, 312 p.

FÉNELON (P.), 1971. - La Loire : crues et embâcles, Tours, Nouvelles Éditions latines, 111 p.

GiRAudet (A.), MÉnANTEAu (L.), 2001 - « Les eaux de la Loire dans la région d'Ancenis : chronique événementielle », Histoire et Patrimoine au Pays d'Ancenis, ARRA, n 16, p. 5-18.

GUILLON (A.), 2003. - « Le risque d'inondation par débordement de rivière ", Cahiers de l'IAURIF, $\mathrm{n}^{\circ}$ 138 , p. $48-63$.

GinSBURGER-VOGEL (M.), 2004. - « D’un seuil à l'autre... Le seuil de Bellevue : légendes et réalité », La Loire et ses terroirs, $\mathrm{n}^{\circ} 49$, p. 12-18.

JEANNEAU (J.), 1984. - « Les grandes crues de l'hiver 1982 et du printemps 1983 », Norois, vol. 31, n 124, p. 617-618.

Jousseaume (V.), Croix (N.), Mercier (D.), 2003. - La Chapelle-Basse-Mer : commune ligérienne, guide de géographie locale, Rennes, Presses universitaires de Rennes, $96 \mathrm{p}$.

JoYeux (E.), 2004. - L'évaluation de la vulnérabilité du territoire agricole du val de la Divatte face au risque d'inondation de la Loire, mémoire de DEA, université de Paris 8 (en cours). 
LANDREIN (J.), 2003. - Le risque d'inondation à La Chapelle-Basse-Mer commune du Val nantais, mémoire de Maîtrise, Université Paris 4 Sorbonne, 277 p.

LE Guillou (F.-A.), 2004. - La construction dans le Val nantais 1990-2003, mémoire de Maîtrise, Université de Nantes (en cours).

LEMARTINEL (B.) (coord.), 2001. - Au chevet d'une catastrophe. Les inondations des 12 et 13 novembre 1999 dans le sud de la France, Perpignan, Presses universitaires de Perpignan, 198 p.

MARION (G.), 1981. - La levée de la Divatte, mémoire de Maîtrise, université de Nantes, 168 p.

MASSON (M.), GARRY (G.), BALLAIS (J.-L.), 1996. - Cartographie des zones inondables. Approche hydrogéomorphologique, Paris La Défense, éditions Villes et territoires, $100 \mathrm{p}$.

Mercier (D.), 2004. - « La mémoire des crues dans le Val nantais », La Loire et ses terroirs, $\mathrm{n}^{\circ}$ 49, p. 19-23.

MeUnier (T.), 2003. - Le risque d'inondation dans le Val nantais. L'exemple de Saint-Julien-de-Concelles, mémoire de Maîtrise, université Paris 4 Sorbonne, 215 p.

Oudot (D.), 1989. - « Les îles de la Loire angevine armoricaine entre les Ponts-de-Cé et Montjeansur-Loire ", Norois, vol. 36, n 142, p. 205-223.

PotTIER (N.), 1998. - L'utilisation des outils juridiques de prévention des risques d'inondation : évaluation des effets sur l'homme et l'occupation des sols dans les plaines alluviales (application à la Saône et à la Marne), thèse, École Nationale des Ponts et Chaussées, 594 p.

-, 2002. - « Gestion du risque d'inondation et maîtrise de l'urbanisation dans le Val de Saône ", dans J.-P. Bravard, J. Combier, N. Commerçon (dir.), La Saône, axe de civilisation, Lyon, Presses universitaires de Lyon, p. 197-213.

Rode (S.), 2001. - « Des inondations et des hommes. Représentation et gestion territoriale du risque d'inondation dans trois communes du Val de Loire (Saint-Pierre-des-Corps, La Riche, Bréhémont) », Grafigéo, n 14, 142 p.

SCHUlÉ (C.-A.), 2000. - « Les situations critiques dans la gestion de la Loire et de son bassin », dans Bravard (J.-P.) (dir.), 2000. - Les régions françaises face aux extrêmes hydrologiques. Gestion des excès et de la pénurie, Paris, Sedes, p. 232-246.

VARAGNe (M.), 2003. - Le jour où la Loire débordera..., Romorantin, Communication-Presse-Édition, $224 \mathrm{p}$.

VINET (F.), 2003. - Géographie des risques. Crues et inondations dans la France méditerranéenne. Les crues torrentielles des 12 et 13 novembre 1999 (Aude, Tarn, Pyrénées-orientales, Hérault), Nantes, Éditions du Temps, $224 \mathrm{p}$.

\section{NOTES}

1. Un pavé IRIS-2000 est un espace défini par l'INSEE comme un "petit quartier» dont la population est comprise entre 1800 et 5000 habitants. Les données des RGP 1999 et 1990 peuvent être obtenues sous ce découpage.

2. Dans l'Ouest de la France, le chef-lieu de commune se nomme le bourg et les écarts se nomment les villages.

3. Cf. Ouest-France du 26 février 2004 et Presse-Océan du 10 mars 2004. 
4. Il aurait été intéressant d'inclure dans cette observation, la commune de Basse-Goulaine, à l'aval du Val nantais, dont le maire est également député et, compte tenu de son appartenance à la majorité parlementaire, a participé à l'élaboration et au vote des lois de 1995 et de 2003.

5. Sur les projets issus du plan Loire Grandeur Nature, voir le site : http://www.loire-estuaire.org

6. Une évaluation économique de l'occupation du Val nantais est en cours (Joyeux, 2004).

\section{RÉSUMÉS}

Le Val nantais, qui était une zone d'expansion des crues de la Loire, a été protégé par une digue au milieu du XIx ${ }^{\mathrm{e}}$ siècle. Puis, durant toute la seconde moitié $\mathrm{du} \mathrm{xx}^{\mathrm{e}}$ siècle, il a été asséché jusqu'à devenir un bassin de production maraîcher. Aujourd'hui, cet espace aménagé par et pour l'agriculture, est convoité pour l'urbanisation du fait de l'expansion de l'aire urbaine nantaise. L'accroissement récent de la vulnérabilité des biens et des personnes dans le Val nantais met en évidence les conflits d'intérêts entre les individus et la collectivité, entre le local et le national. Cet exemple pose concrètement la question de l'application des politiques nationales à l'échelle communale.

The Val nantais, which is the flood plain of the Loire River, has been protected from the waters by a levee in the middle of the $19^{\text {th }}$ century. Since then, it has been drained by agriculturists with the support of the local municipalities, in order to create an important vegetables production area. The Val nantais lies 20 kilometers upstream Nantes City, and since the 1970's it is within reach of the urban extensions. Therefore human and economic vulnerability to floods have strongly increased. This situation rises the issue of national policy application at local scale.

\section{INDEX}

Mots-clés : agriculture, aménagement foncier, inondation, peuplement, politique locale, politique nationale, risque naturel, urbanisation

Index géographique : France, Loire (fleuve et vallées), Loire-Atlantique, Nantes

Keywords : government policy, inundation, land management, local policy, natural hazards, settlement

\section{AUTEURS}

\section{VALÉRIE JOUSSEAUME}

Institut de géographie et d'aménagement régional - Université de Nantes, Espaces géographiques et sociétés, UMR 6590 - CNRS, valerie.jousseaume@humana.univ-nantes.fr 


\section{JULIE LANDREIN}

UFR de géographie et aménagement - Université Paris 4 Sorbonne, Dynamiques et évolutions des paysages atlantiques et méditerranéens, EA 2579, Géodynamique des milieux naturels et anthropisés, UMR 6042 - CNRS

\section{DENIS MERCIER}

UFR de géographie et aménagement - Université Paris 4 Sorbonne, Dynamiques et évolutions des paysages atlantiques et méditerranéens, EA 2579, Géodynamique des milieux naturels et anthropisés, UMR 6042 - CNRS, denis.mercier@paris4.sorbonne.fr 